
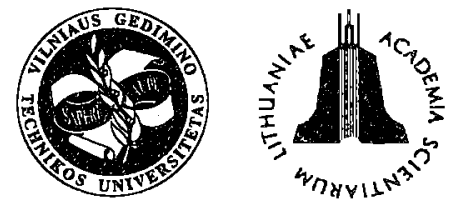

ISSN 1648-4142 TRANSPORT

http:/www.vtu.It/english/editions

\title{
THE VIBROACOUSTICAL RESEARCH OF ROLL BEARINGS DEFECTS
}

\author{
Eimantas Juodzevičius \\ Dept of Automobile Transport, Vilnius Gediminas Technical University, J. Basanavičiaus g. 28, \\ LT-2009 Vilnius, Lithuania. Tel. (85) 2126925.E-mail: jeimantas@one.lt
}

Received 200207 01; accepted 20021221

\begin{abstract}
This article presents the description, results and conclusions of the methodology of the vibroacoustical research of the transmission of one of the units of a tactical car - of the bevel bearings of the main gear reducer. To explore the vibroacoustical research of the roll bearings with reference to the latest western literature the main diagnostic methods are used. Their advantages and disadvantages, the limits of the vibration frequencies, where the assessment of acoustical parameters of vibration, are analyzed. The defects of the bearings are identified exploring the level of the noise intensiveness in the proximate points from the bearings, components (changing the diametric clearance), and the results are presented in the form of the spectra of the amplitude surrounding.
\end{abstract}

Keywords: vibroacoustical, bearing; transmission; level of noise; diagnostic; abrasion; analysis of vibration.

\section{Introduction}

Presently worldwide producing, exploiting either tactical cars or those of other branches of economy and their units, credibly operating units are being intensively created which could be controlled without dismantling; ways of localization of defects are being searched for with the purpose of identification of their technical state.

The components of bearings, which are an indispensable part of modern cars and mechanisms, often limit the resources of the latter. As a rule, there is no convenient access to the components of bearings to exercise technical maintenance and to identify breakdowns. During the exploitation great attention is paid to the creation of diagnostic methods without dismantling, in the first place - vibroacoustical [1,2], the more so because the reliability of the roll bearings depends on their vibration and production precision [3].

The nature of the bearing breakdowns in the exploiting conditions is divided into the following groups [4]:

1. Breakdowns for the metal fatigue;

2. Increased abrasion;

3. Breakdowns, which appeared having changed clearances and tightness between bearing clamps and shafts, bearings, seats;

4. Defects for poor lubrication.

The article presents the methodology, equipment gathering, results and conclusions of the vibroacoustical research of one of the units of the transmission of a tactical car - roll bearings of the main gear reducer (the run $\sim 42000 \mathrm{~km}$ ). This reducer has been selected not incidentally - roller bevel and simple, adjusting and misadjusting bearings are assembled in it. When there are only roller bearings, it is easier to compare the received spectra of the noise intensiveness level of various bearing components because the reasons of the noise formation are the same [5]. Physically two principal reasons of noise formation can be distinguished: friction of touching parts and forces of unbalanced inertia (these forces are trivial in bearings), which appear when the parts of the mechanism (component) are moving. In some cases dynamic shocks of parts of the mechanism are distinguished but this factor is attached to the operation of the inertia forces.

During the testing the attention will be focused on the two references - the potentials of the vibroacoustical equipment and the dependence of the assembled in the reducer worn and replaced new bearings, in the bearings adjusted by the clearance to the level of spread noise intensiveness. Thus, the purpose of the research is:

- to identify the level of spread noise intensiveness of old and new turning bearings;

- to identify the effect of the change of the clearance in the adjusting bearings on the level of noise intensiveness.

\section{The Vibroacoustical Diagnostic Methods of Roll Bearings}

Having analysed diagnostic methods presently applied by English [5], Danish [2] scientists of acoustic sphere, conditionally they can be divided into three groups. Those methods, which are based on the detachment and analysis of discreet components of the bearing oscillations of the forced or principal excitation frequencies belong to the first group. The main frequencies of excitation of bearing oscillations are the following [5]:

the turning frequency of the settler 


$$
f_{\text {sep }}=\frac{n}{2}\left(1-\frac{d}{D} \cos \beta\right)
$$

the turning frequency of roll bodies

$$
f_{r k}=\frac{n D}{2 d}\left[1-\left(\frac{d}{\bar{D}}\right)^{2} \cos ^{2} \beta\right]
$$

the frequency of repetition of roll bodies in the outside ring of the bearing

$$
f_{i \check{s}}=z \frac{n}{2}\left(1-\frac{d}{D} \cos \beta\right) ;
$$

the frequency of repetition of roll bodies in the inside ring of the bearing

$$
f_{v}=z \frac{n}{2}\left(1+\frac{d}{D} \cos \beta\right),
$$

here, $n$ - the frequency of turning of the shaft, $s^{-1} ; d$ - the diameter of the bearing roll; $D=\left(D_{i}+D_{\mathrm{o}}\right) / 2$ - the diameter of the circle, going through the centres of the roll bodies; $D_{i}$ and $D_{\mathrm{o}}$ - the diameters of the inside and outside rings of the bearing, respectively; $\beta$ - the angle of the contact, ${ }^{\circ} ; z$ - the number of the rolls.

The methods based on the analysis of the frequencies evoked by defects of various bearing elements also belong to the first group. With the help of these methods it is possible to identify the place of damage in a bearing (outside, inside rings, settler or rolls). The diagnostic features of bearing defects can be characteristics of signals, coinciding with the frequency of roll of balls or rolls on damaged surfaces: the amplitude of the signal, the relation of the signal energy with the level of noise, the components of spectral amplitudes with the frequency of repetition of signals, the detachment of composite frequencies. Spectral and correlation methods, analysis methods with octave or other filters synchronically meshing with turning frequency are used for the identification of these signals from a complex vibroacoustical signal.

The diagnostic methods of all bearing components are attached to the second group. During the exploitation the breaking down process of bearing components often consists of several defects of bearing elements. In that case the breaking down process manifests itself by the change of amplitudes in spectrograms, changing the amplitudes of many components. Bearing diagnostic method based on statistical vibration analysis is also used. Here statistical vibration - excess assessment in four frequency ranges of a bearing is exercised: $3-5 \cdot 103 \mathrm{~Hz} ; 5-10 \mathrm{kHz}$; $15-20 \mathrm{kHz}$.

When there is no defect the excess equals to zero in all frequency bands; a trivial damage changes parameters in low frequency bands, greater damages influence high frequencies, restoring the excess to the primary low frequency sphere. This method, inter alias, may be used for the prognostication of bearing resources [4]. The advantage of the method is that it is insusceptible of changes of speed of unsound bearing and load when it is turning.

The third group of diagnostic methods consists of the analysis of oscillation in resonant frequency. The most perspective are considered those methods with the help of which the defects, which appear during the exploitation, are identified. They are based on the analysis of the change of characteristics of acoustical signal amplitude component of a bearing resonant frequency $\left(0,5 \mathrm{kHz}<f_{r}\right.$ $<20 \mathrm{kHz}$ ). In this case the identification of defects of roll bearings is based on the theory of impact of the mechanical system of consistency of impact signals on the resonant frequency. Performing spectral analysis of bearing vibration in low frequencies, camouflaging spectral effect of forced and own fluctuations of a component, which does not allow showing up the components that characterize a bearing defect, is observed. The single restriction applying the application of impact signals in the presence of low frequencies is exclusive vibration measurement, i.e. a vibrosensor is assembled straight on a bearing hoop.

In the zone of high frequency resonance suitably precise results are gained measuring noise intensiveness [6]. When a bearing is turning in resonant frequency the spectral analysis of the amplitude component of acoustic signal is the most convenient one for measurement, the analysis of the results and interpretation. In this case diagnostic characteristics may be frequency values of repetition of defects (1) - (4) (when a bearing is turning) or other informative parameters.

\section{Measurement Equipment and Methods}

The measurements were performed using the equipment made by Danish firm Bruel \& Kjaer in 1991 - 1993. The equipment consisted of:

- $\quad$ Sound Intensity Analyser Type 2034;

- $\quad$ Sound Intensity Probe Type 3519;

- Microphones of Probe Type 4178;

- Digital Recorder Type 7400;

- Telltale Package WT 9124;

- Graphic Literal Printer Type 2313;

- PC.

This system constitutes a keyboard frequency analyser that measures sound intensity and sound pressure levels in mono-octave and trio octave bands, when the frequency is $3,2-10 \mathrm{kHz}$ (sound intensity) and 1,6$20 \mathrm{kHz}$ (sound pressure). The results are presented on the display screen according to real - time analysis principle, where inter alias array direction of sound intensity is indicated, and Printer 2313 prints the results.

One of the driving bridges of BTR-60 PB was removed from the car and, with the purpose of setting operational conditions to those of exploitation, through tough fastening elements it was embedded on an immobile stand. Two measurements of the seven points in the horizontal 
plane of the principal gear were performed, when a driving tooth wheel was turning at the frequency of $2000 \mathrm{~min}$ 1 : when its output $-42000 \mathrm{~km}$ and having replaced driving, driven tooth wheels as well as roller bearings by new ones. The distance from intensymmetrical probe to the surface of sound resource was $60 \mathrm{~mm}$, except the third and the nifth position; the distance from the under part of the unit to the floor is $550 \mathrm{~mm}$. The measurement scheme is presented in Fig 1.

The oil in the reducer according to the producers' instructions has been in exploitation; the level is according to the norms determined in the instructions. The unit is fully completed, regulated and there are no breakdowns preventing it from normal functioning. The revolutions of the driving shaft were set and corrected by an independent tachometer with stroboscope lamps.

\section{Measurememe Procedare}

Having analysed the main vibroacoustical diagnostic methods (Section 2), we choose the third group of methods, which matches according to our available equipment (Section 3). The analyser, with reference to the set frequency on his display, presents the spectra and peaks of noise intensiveness filtrated in $1 / 3$ octave band, and amplitudes.

Although measurements are performed in a closed room the reverberated noise intensiveness does not have any impact on the results of measurements because the probe of 3519 Type traces changes of noise in one direction only [2].

For good results well - defined measurement conditions are required. Information on the references used, environmental conditions, the grid size, presence of a reflecting plane, etc. are entered into a measurements set up (register) and stored with the measured data.

Before performing any calculation the data are inspected and validated using the functions available in the software to compare the directly measured data with the results derived from the description of the sound field established by STSF program. Comparison is needed here in order to obtain accurate values of references [2].

If necessary, data can be re - measured and replaced. There will always be some discrepancy between the directly measured and the caiculated spectra due to non stationary of the source during the measurement at low frequencies, the use of an insufficient set of references, or uncorrelated background noise. These errors during this measurement are not estimated.

\section{Measurement Results}

In order to identify prevailing vibration frequencies of the principal gear with worn bcarings we perforn measurements at all seven points, when the shaft of the reducer is turning $2000 \mathrm{~min}^{-1}$ (Fig 1). The results of the measurement of noise intensiveness are presented in Fig 2.

While analyzing the diagram, presented in Fig 2, we see that the greatest noise intensiveness is when the frequency is $400 \mathrm{~Hz}$. Incidentally, the maximum level of noise intensiveness $(50 \mathrm{~dB})$ is at the seventh point (the bearing of the left axle shaft. Having replaced the roll bearings by new ones and having performed parallel measurements, such results are obtained (Fig 3). While analyzing this diagram, we notice that the greatest noise intensiveness is when the frequency is also $400 \mathrm{~Hz}$ but its total average level is $3 \div 3,5 \mathrm{~dB}$ lower than during the first measurement.

With the purpose to identify bearing defects levels of noise intensiveness are analyzed at the third, fourth and seventh points (Fig 1), when the frequency is $400 \mathrm{~Hz}$. Those measurement points are selected that are the proximate to the bearings (the impact of the noise spread by adjacent spheres is escaped) and the level of noise of which is the greatest. Fig 4 presents the spectra of amplitude component of those points.

In the spectrum of the surrounding the maximum and harmonic peaks of the frequency of turning of bearings are clearly seen. At the seventh point the level is lower because the bearing turns almost twice more slowly.

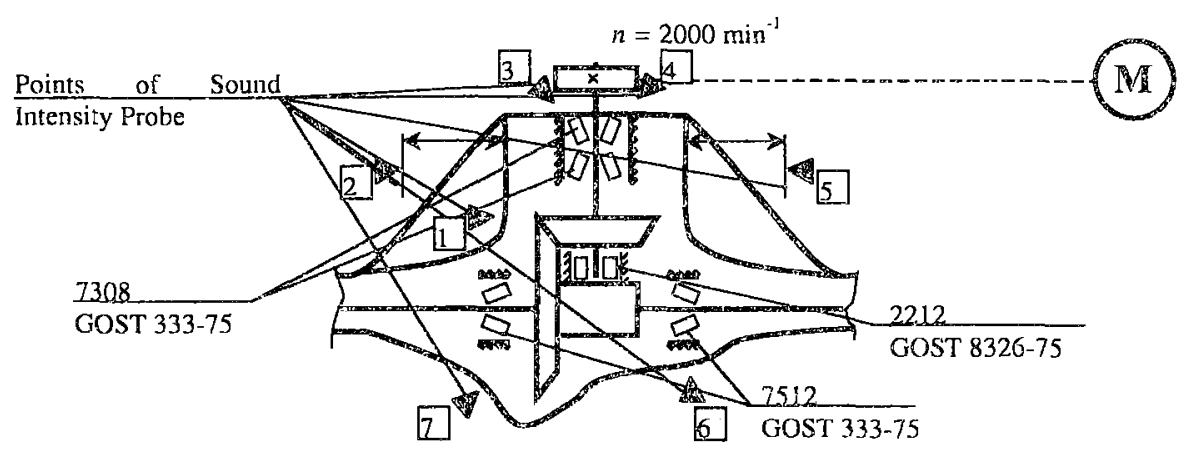

Fig 1. The kinematic scheme of the principal gear of the reducer with roll bearings and the positions of the intensymmetrical probe $(1 \div 7)$ when measurements are performed 


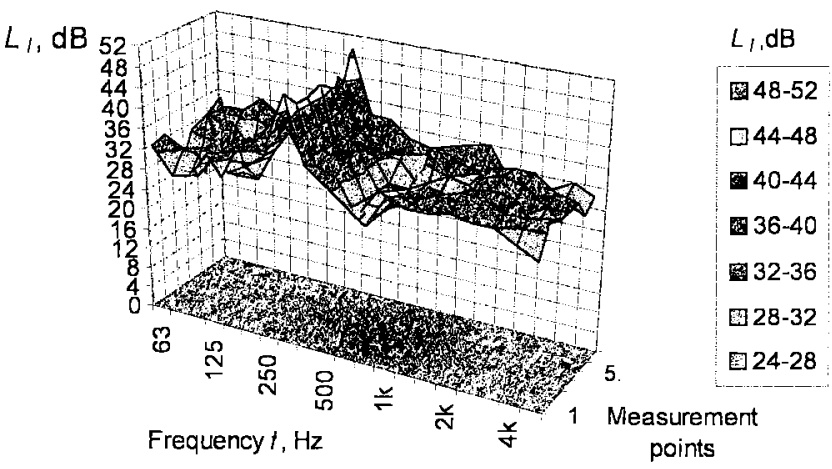

Fig 2. The levels of noise intensiveness $L_{1}$ of the reducer of the principal gear $1 \div 5 \cdot 10^{3} \mathrm{~Hz}$ in the frequency spectrum

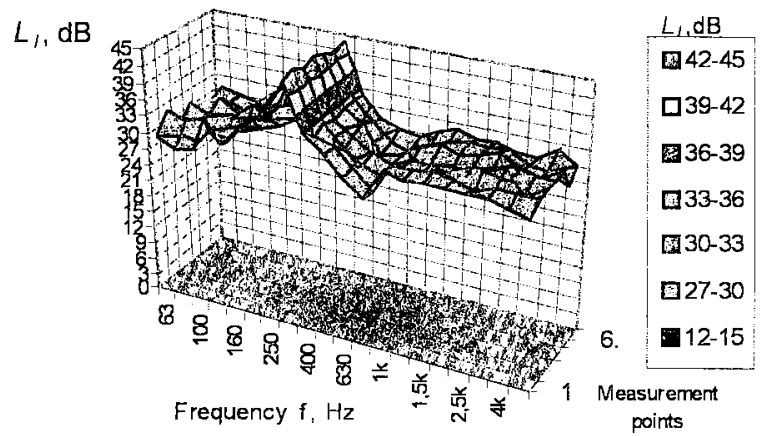

Fig 3. The levels of measurement of noise intensiveness $L_{1}$ of the reducer (properly assembled and regulated) of the principal gear $1 \div 5 \cdot 10^{3} \mathrm{~Hz}$ in the frequency spectrum

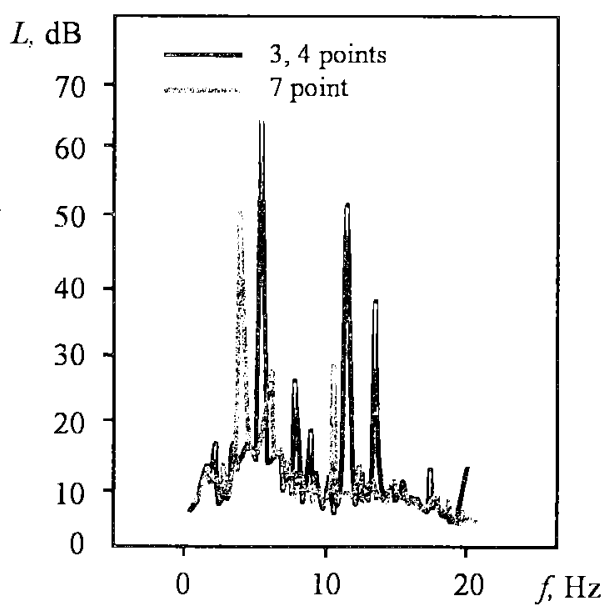

Fig 4. The spectra of the amplitude surrounding of noise intensiveness of the bearings of the reducer of the principal gear, when the bearings are worn $(400 \mathrm{~Hz})$

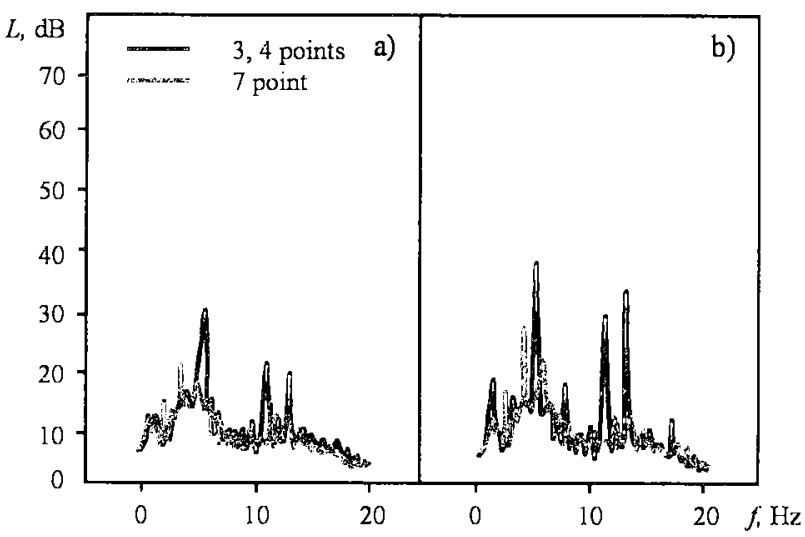

Fig 5. The spectra of the amplitude surrounding of noise intensiveness of the bearings of the reducer of the principal gear, when the frequency is $400 \mathrm{~Hz}$; a - of new bearings; $b$ of new bearings, having left a diametric clearance of $250 \mu \mathrm{m}$

Fig 5 presents the spectra of amplitude surrounding of noise intensiveness of the reducer with properly regulated new bearings and with the diametric clearing.

Analyzing the diagrams presented in Fig $5 \mathrm{a}$ and $\mathrm{b}$, we see that in new bearings the average level of noise intensiveness is $22 \mathrm{~dB}$ (Fig 5 a), and the maximum peaks reach only $30 \mathrm{~dB}$. Moreover, the same as the situation with old bearings, for the reasons mentioned above, the noise is greater at the third and fourth points. The bearings 7512 and 7308 (GOST 333-75) in the tested reducer are regulated. In the mentioned bearings, having left the diametric clearing of $250 \mathrm{~mm}$, the average level of noise intensiveness is also about $22 \mathrm{~dB}$ but the maximum peaks reach $40 \mathrm{~dB}$ (Fig 5 b)

After comparing Fig $5 \mathrm{~b}$ with Fig 4 it is not difficult to notice that the level of noise intensiveness of the new bearings with a clearance is lower than that of the normally regulated old ones. The conclusion may be drawn that there is a defect of surface of roll bodies or bearing rings in the bearings.

Having dismantled the bearings, a clear carbonization nick could be noticed with the naked eye on the inside and outside rings.

\section{Conclusions}

1. Turning the driving shaft of the principal gear at the revolutions of $2000 \mathrm{~min}-1$, the identified prevailing vibration frequency of the reducer is $400 \mathrm{~Hz}$.

2. When the output of the bearings is $42000 \mathrm{~km}$, the measured average level of noise intensiveness is $3 \div 3,5$ $\mathrm{dB}$ greater than this of the new ones and the maximum peaks are $100 \%$ greater.

3. In the bearings regulated by the tested reducer, having left the diametric clearance of $250 \mathrm{~mm}$, the average level of noise intensiveness is $22 \mathrm{~dB}$ but the maximum peaks reach $40 \mathrm{~dB}$, that is, they are $20 \mathrm{~dB}$ less than those in properly regulated old bearings. 
4. It was ascertained that when the components of the bevel bearings spread greater level of noise intensiveness than $40 \mathrm{~dB}$; it is necessary to check the adjustment of bearings or to replace them.

\section{References}

1. Watkinson, P. S. Sound intensity measurement. The shock and vibration digest, Vol XVII, No 5, 1998, p. 42-48.

2. Hald, J. STSF unique technique for scan-based near field acoustic holography without restriction on coherence. Bruel \& Kjaer. Technical Review, No 2, 1993.40 p.
3. Stobener, U. \& Lothar, G. Surveys of rotating machineryusing vibration monitoring techniques. Norwegian Shipping News, No 11, 1997, p. $25-29$.

4. Genkin, M. D.; Sokolova, A. G. and other. Introduction of Acoustics Dynamic of Machines. M.: Nauka, 1984. 285 p.

5. Eibeheiry, E. M. Effects of small travel speed variation on active vibration control in modern vehicles. Journal of Sound and Vibration, Vol 232, No 5, 2000, p. 857-875.

6. Cheng, C. C. \& Shiu, J. S. Transient vibration analysis of a high-speed feed drive system. Journal of Sound and Vibration, Vol 239, No 3, 2001, p. 489-504. 